\title{
Síndrome de Carney con mixoma mamario: un tumor muy infrecuente de manejo interdisciplinario
}

\author{
Paolo Ricci A. ${ }^{1}$, Álvaro Ibarra $V^{2}$ \\ ${ }^{1}$ Unidad de Ginecología, Departamento de Obstetricia y Ginecología, ${ }^{2}$ Departamento de Anatomía Patológica. \\ Clínica Las Condes. Santiago, Chile.
}

\section{RESUMEN}

Reportamos caso clínico de una mujer de 22 años quien presentó un fibroadenoma mixoide de localización mamaria. Previamente se había diagnosticado un microadenoma pituitario y tiroiditis con bocio difuso leve. Se realizó un estudio multidisciplinario para descartar otras localizaciones de tumores mixoides en el contexto del infrecuente síndrome de Carney.

\section{PALABRAS CLAVE: Mixoma mamario, fibroadenoma mixoide, tumor mesenquimático de mama, complejo de Carney}

\section{SUMMARY}

We report a 22-year-old woman, who presented a myxoid fibroadenoma affecting the breast. Previously a pituitary microadenoma and thyroiditis with mild diffuse goiter was diagnosed. A multidisciplinary study was performed to rule out the location of other myxoid tumors in the context of infrequent Carney syndrome.

\section{KEY WORDS: Breast myxoma, fibroadenoma myxoid, mesenchymal tumor of the breast, Carney complex}

\section{INTRODUCCIÓN}

Los mixomas son tumores de origen mesenquemático y comportamiento benigno. Pueden afectar diferentes partes del cuerpo. Si están asociados a pigmentación de piel e hiperactividad endocrina, forman parte del síndrome de Carney $(1,2)$. Este síndrome fue descrito por primera vez en 1985, y se han informado unos 150 casos, afectando en igual frecuencia a ambos sexos. Su diagnóstico mayoritariamente es realizado entre los 10 y 20 años (3). Las manifestaciones endocrinas más frecuentes son la acromegalia, las producidas por tumor testicular y tiroideo, y el síndrome de Cushing debido al compromiso por tumor adrenocortical (4). Algunas localizaciones de mixomas son: el corazón (donde generalmente son múltiples y se asocian a letalidad), riñón, boca, nariz, ojos, piel y mucosas (5). Entre las menos frecuentes se encuentra la mama $(6,8)$.

Este síndrome es de transmisión genética, autosómica dominante (9). En la mayoría de los pacientes se produce una mutación del gen PRKA$R 1 A$, que codifica la subunidad reguladora R1alfa, del AMP cíclico dependiente de la protein-quinasa $A$, lo que se conoce como síndrome de Carney tipo I (10). Este gen esta ubicado en el cromosoma $2 \mathrm{p} 16$. Sin embargo, se han estudiado algunos casos que no tiene esta ubicación comprometida, por lo que existiría un segundo locus que podría estar afectado, lo que recibe el nombre de síndrome de Carney tipo II (11). Durante estos dos últimos años, 
se publicó una nueva mutación en la subunidad reguladora de la proteína quinasa A (IVS2-1G, mutación del empalme en el intrón 2), en una paciente con hijos gemelos, en que uno de ellos es portador de la misma alteración (12,13). En general, el 70\% de los casos que tienen síndrome de Carney, son familiares.

Presentamos un caso de síndrome de Carney, con presentación mamaria como fibroadenoma mixoide, asociado a microadenoma hipofisiario y bocio difuso heterogéneo. Se realizó un estudio multidisciplinario para descartar otras localizaciones de tumor mixoide.

\section{Caso clínico}

Mujer de 22 años, con antecedente de controles por tiroiditis crónica con un bocio difuso leve, diagnosticado durante el 2008. Además durante ese mismo año se realizó una resonancia magnética de hipófisis, como parte del estudio de cefalea, que evidenció la presencia de un microadenoma hipofisiario.

Durante este tiempo consulta en un control ginecológico por la auto detección de dos tumores mamarios izquierdos. Uno estaba ubicado en la unión de los cuadrantes superiores de $3 \mathrm{~cm}$ y el otro en el cuadrante superior externo de $2 \mathrm{~cm}$. Además, otro tumor en la mama derecha de $1 \mathrm{~cm}$. El estudio ecográfico mamario evidenció la presencia de imágenes clasificadas como Bi-rads IV. Se realizó una tumerectomía con biopsia rápida y diferida, que demostró la presencia de fibroadenomas, uno de ellos mixomatoso (Figura 1 y 2 ).

Estudio postoperatorio: Se realizó control y estudio multidisciplinario, descartando la presencia de otras localizaciones de tumores mixoides. Fue controlada en conjunto, por cardiólogo, dermatólogo,

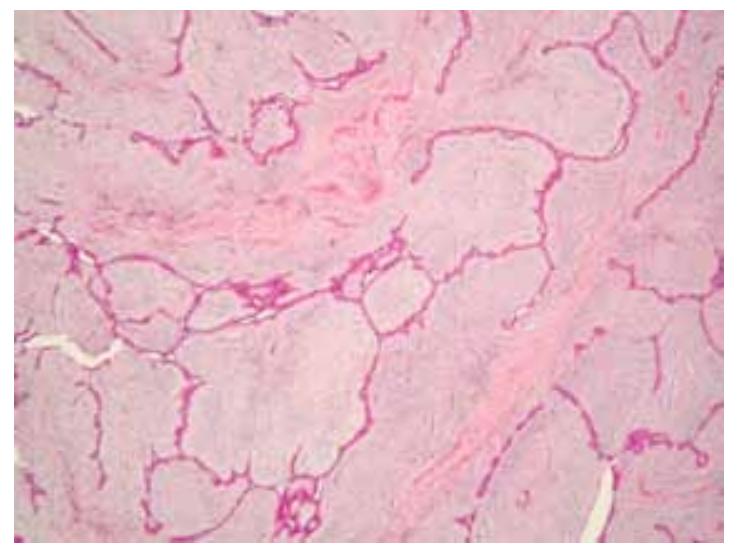

Figura 1. Tinción Hematoxilina-Eosina. Visión 4x. Se observa fibroadenoma con patrón intracanalicular con estroma extensamente mixoide.

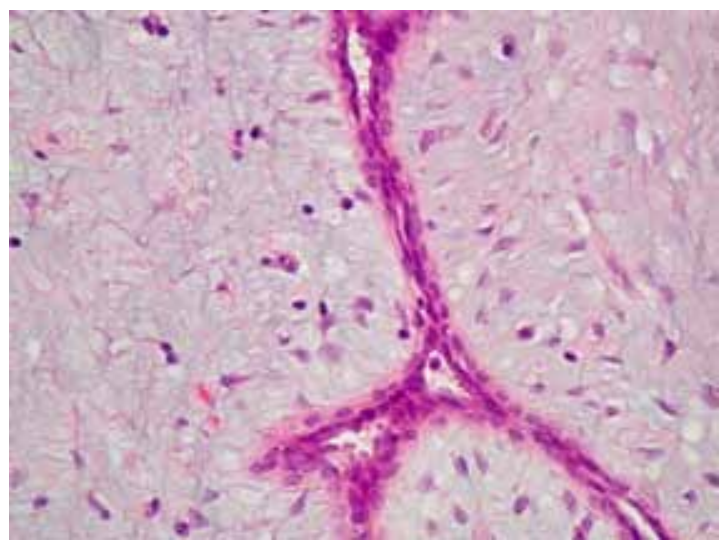

Figura 2. Tinción Hematoxilina-Eosina. Visión 40x. Detalle de la histología que muestra el tejido fibroconectivo laxo con células estromales sin atipias. Fibroadenoma mixoide.

otorrinolaringólogo, ginecólogo, cirujano, oncólogo y genetista. Se realizó ecocardiograma $2 D$, ecografía abdominal y pelviana, que resultaron normales. La ecografía tiroidea demostró un bocio leve difuso heterogéneo. La tomografía computada multicorte de abdomen y pelvis resultó normal. En la tomografía de cavidades perinasales se encontró una formación polipoídea del seno maxilar que correspondió a un quiste de retención.

Durante el 2009, se detectó un nuevo nódulo en mama izquierda de $1 \mathrm{~cm}$, en el cuadrante superointerno, Bi-rads $4 \mathrm{~A}$. La biopsia core bajo ultrasonido demostró que se trataba de un fibroadenoma sin atipias ni signos de malignidad. Actualmente se encuentra en controles y seguimiento anual, sin variaciones.

\section{DISCUSIÓN}

El término mixoma fue descrito por primera vez por Virchow, para describir tumores que se asemejaban a la estructura del cordón umbilical (14). Fue Stout quien más tarde describe el mixoma como una neoplasia verdadera, que se caracteriza por la presencia de células estrelladas en un estroma laxo o mixoide, y lo consideró como un tumor del mesénquima primitivo (15).

Los mixomas encontrados como tumor único, aislado de mama, sin formar parte de un síndrome de Carney, son extremadamente raros. No hay más de 9 casos en la literatura internacional, reportados entre 1915 y 2010 (16-19). Para clasificar a una paciente como portadora de un síndrome de Carney, debe cumplir con 2 o más de las siguientes condiciones: (1) mixoma cardíaco, (2) mixoma cutáneo, (3) mixoma mamario, (4) pigmentación muco-cutánea irregular, enfermedad nodular pigmentaria primaria 
adrenocortical (síndrome de Cushing), tumor testicular (precocidad sexual), (7) adenoma pituitario secretor de hormona del crecimiento (acromegalia/gigantismo) (20). En este caso existía el diagnóstico de un microadenoma hipofisiario y una tiroiditis con bocio leve difuso. Se descartó la presencia de pigmentación mucocutánea, que habitualmente se expresa como lentigos y nevus de color azul. Estos se deben a la presencia de schwanoma melanótico psamomatoso y a nevus epiteliales azules, respectivamente, que se encuentran principalmente en extremidades y tronco, menos frecuente en cabeza y cuello (21).

Es muy importante, una vez que se ha encontrado un mixoma, realizar un seguimiento para descartar la aparición de otras localizaciones, ya que puede tratarse de un síndrome de Carney. Un reporte realizado en 1999, describió un caso de pólipo nasal, que correspondía a un tumor mixoide (22). En el caso que presentamos se encontró una formación polipoídea, que correspondió a un quiste de retención. Tal como fue en este caso, se debe descartar la presencia de mixomas en zonas muco-cutáeas como párpados, orejas, cavidad bucal y nasal, vulva, en pene en hombres y toda la piel. Por esta razón es importante el trabajo de un equipo multidisciplinario, que además descarte una eventual hiperactividad endocrina y la presencia de mixomas en órganos, como corazón. Esta última localización es la más frecuente, con una mortalidad de hasta el $25 \%$, principalmente debido a embolias. Debido a su alta letalidad, especial interés debe tener el estudio cardíaco (22).

En pacientes con síndrome de Carney, el estudio de la mama con mamografía, ultrasonido o resonancia magnética, puede demostrar la presencia de tumores, que en la gran mayoría de las veces corresponden a fibradenomas mixoides $(22 \%)$. Son el reflejo de la predisposición al crecimiento mixoide y que no aumenta el riesgo de cáncer, por tratarse de un tumor benigno (23). Sin embargo, debemos tener presente, que en la literatura internacional existe un reporte de recurrencia local en cuatro ocasiones, con aparición de un liposarcoma en la tercera y un mixosarcoma en la cuarta (24).

\section{CONCLUSIÓN}

El mixoma mamario es un tumor mesenquimático, de carácter benigno, de muy baja frecuencia. Generalmente se encuentra formando parte del síndrome de Carney, que puede afectar diferentes partes del cuerpo. Frente al hallazgo de un fibroadenoma mixoide debe promoverse un estudio completo y multidisciplinario, para descartar otras localizaciones. Existen pocos casos informados como tumor mixoide aislado de la mama. Al encontrarse un mixoma aislado, debe realizarse un seguimiento de la paciente, para detectar la posible aparición en otro lugar.

\section{REFERENCIAS}

1. Bertherat J. Carney complex (CNC). Orphanet J Rare Dis 2006;1:21.

2. Zahedi RG, Wald DS, Ohri S. Carney complex. Ann Thorac Surg 2006;82:320-2.

3. Carney JA, Gordon H, Carpenter PC, Shenoy BV, Go VL. The complex of myxomas, spotty pigmentation, and endocrine overactivity. Medicine (Baltimore) 1985;64:270-83.

4. Groussin L, Cazabat L, René-Corail F, Jullian E, Berthrrat J. Adrenal pathophysiology: lessons from the Carney complex. Horm Res 2005;64:132-9.

5. Jellis $C$, Hunter A, Sutton R. Multimodal imaging of an atrial myxoma. Cardiovasc Pathol 2009;18:379-80.

6. Balci P, Kabakci N, Topcu I, Canda T, Güray M, Ozfidan S. Breast myxoma: Radiologic and histopathologic features. Breast J 2007;13:88-90.

7. Wee A, Tan CE, Raju GC. Nerve sheath myxoma of the breast. A light and electron microscopic, histochemical and immunohistochemical study. Virchows Arch A Pathol Anat Histopathol 1989;416:163-7.

8. Carney JA, Toorkey BC. Myxoid fibroadenoma and allied conditions (mixomatosis) of the breast. A heritable disorder with special associations including cardiac and cutaneous myxomas. Am J Surg Path 1991;15:713-21.

9. Wilkes D, McDermott DA, Basson CT. Clinical phenotypes and molecular genetic mechanisms of Carney complex. Lancet Oncol 2005;6:501-8.

10. Casey M, Vaughan CJ, He J, Hatcher CJ, Winter JM, Weremowicz S, et al. Mutations in the protein kinasa A R1alpha regulatory subunit cause familial cardiac myxomas and Carney complex. J Clin Invest 2000;106:R31-8.

11. Irvine $A D$, Armstrong $D K$, Bingham EA, Hadden DR, Nevin NC, Hughes AE. Evidence for a second genetic locus in Carney complex. Br J Dermatol 1998;139:572-6.

12. Halázlaki $C$, Takács I, Butz $H$, Patócs $A$, Lakatos $P$. Novel genetic mutation in the background of Carney complex. Pathol Oncol Res 2012;18:149-52.

13. Halázlaki $C$, Takács I, Butz $H$, Patócs $A$, Lakatos $P$. Novel mutation in a patient with Carney complex. Orv Hetil 2011;152:802-4.

14. Virchow R. Cellular pathology as based upon physiological and pathological histology. Ed. Dover Publications Inc, New York, United States. 1971: 525-6.

15. Stout AP. Myxoma, the tumor of primitive mesenchyme. Ann Surg 1948;127:706-19.

16. Román Santamaría J, Moreno Elola MA, López Santana P. Mixoma mamario presentación de un caso. Rev Venez Oncol 2008;20:146-8.

17. Chan YF, Yeung HY, Ma L. Myxoma of the breast: report of a case and ultrastructural study. Pathology 1986;18:153-7.

18. Arihiro K, Inai K, Kurihara K, Takeda S, Khatun N, Kuroi $\mathrm{K}$, Kawami H, Toge T. Myxoma of the breast: report of a case withunique histological and immunohistochemical appearances. Acta Pathol Jpn 1993;43:340-6. 
19. Magro G, Cavanaugh B, Palazzo J. Clinico-pathological features of breast myxoma: report of a case with histogenic considerations. Virchows Arch 2010;456:581-6.

20. Koyano T, Satoh T, Ohtaki N. Familial cases of cutaneous mixomas and spotty pigmentation (Carney's complex). Nippon Hifuka Gakkai Zasshi 1990;100:1047-52.

21. Carney JA, Stratakis CA. Epitheliod blue nevus and psammomatous melanotic schwannoma: the unusual pigmentes skin tumors of the Carney complex. Semin Diagn Pathol 1998;15:216-24.
22. Tanaka Y, Sano K, ljiri R, Tachibana K, Kato K, Terashima K. A case of large cell calcifying Sertoli cell tumor in a child with a history of nasal myxoid tumor in infancy. Pathol Int 1999;49:471-6.

23. Courcoutsakis NA, Chow CK, Shawker TH, Carney JA, Stratakis CA. Syndrome of spotty skin pigmentation, myxomas, endocrine overactivity, and schwannomas (Carney complex): breast imaging findings. Radiology 1997;205:221-7.

24. Rudan I, Rudan N, Sarcevic B. Locally recurring primary myxoma of the breast: an evidence of malignant alteration. Acta Med Croatica 1996;50:209-11. 